\title{
Ultralate cerebral potentials as correlates of delayed pain perception: observation in a case of neurosyphilis
}

\author{
ROLF-DETLEF TREEDE,* WINFRIED MEIER, $\dagger$ KLAUS KUNZE, \\ BURKHART BROMM*
}

From the Institute of Physiology* and Neurological Clinic, $\ddagger$ University Hospital Eppendorf, Hamburg and Neurological Clinic, $\uparrow$ Johannes Gutenberg University, Mainz FRG

SUMMARY Evoked cerebral potentials were investigated in a patient with neurosyphilis, who showed the symptoms of delayed pain perception in the lower limbs: a pinprick to the legs was perceived with a latency of more than one second. After stimulation with $\mathrm{CO}_{2}$ laser radiant heat pulses, evoked cerebral potentials of upper limbs were observed in a latency range comparable to those of healthy subjects, with a negative peak at $250 \mathrm{~ms}$ and a positive peak at $370 \mathrm{~ms}$. In contrast, after application of laser stimuli to body sites with delayed pain perception, latency of the evoked potentials drastically increased with a vertex negativity at $1300 \mathrm{~ms}$ and a positivity at $1420 \mathrm{~ms}$. Evoked potential measurements with conventional electrical stimuli did not show any difference between affected and unaffected body sites, that is, stimulation of the affected body sites did not produce pathological potentials.

Syphilis affecting the spinal cord can lead to different and often intriguing sensory disturbances. One of hem is the delay of pain perception; in such cases the ratency between a noxious stimulus and the expression of its perception as painful has been reported to be up to several seconds. ${ }^{12}$ Although in the era of efficient antibiotic therapy the incidence of neurosyphilis has declined, ${ }^{3}$ we have been able to examine a patient with pathologically delayed pain perception due to neurosyphilis.

Standard clinical electrophysiological methods generally are of little value in such disorders of pain and temperature. ${ }^{45}$ Therefore, besides conventional somatosensory evoked potentials (SEP), we studied cerebral potentials evoked by brief noxious radiant heat pulses produced by a $\mathrm{CO}_{2}$ laser. This technique has repeatedly been shown to be effective in the assessment of functions in the nociceptive pathways. ${ }^{6-8} \mathrm{We}$ applied these stimuli to various sites of the patient's skin in order to compare cerebral responses after stimulation of intact and of disturbed dermatomes.

Address for reprint requests: Dr Winfried Meier, Neurological Clinic, Langenbeck Str 1, 6500 Mainz, Fed Rep Germany.

Received 12 February 1985.

Accepted 13 May 1988

\section{Methods and material}

\section{Case report}

The study was performed in a 48 year old homosexual businessman, who was admitted to the neurological university clinic with a progressive gait disturbance, numbness of the feet and bladder dysfunction, which had begun about 3 months earlier. Neurological examination revealed only slight tactile hyposensitivity and slightly impaired vibration sense in both feet, as well as a gait disturbance with closed eyes and a positive Romberg sign. Tendon reflexes of quadriceps and triceps surae muscles were lost. Sense of joint position in lower and upper limbs was intact. Sensitivity and reflexes in the upper limb were normal; no cranial nerve disorders or Babinski sign were observed. The most impressive sensory symptom was the delay in pain perception in the lower limb: a single pin prick led to a painful sensation not before one second. Repeated stimulation of about $1 / \mathrm{s}$ lead to an increasing burning and dull pain. This symptom was present in all dermatomes below L2.

Serological tests revealed positive results from Venereal Disease Research Laboratories (VDRL) test, as well as for $T$. pallidum haemagglutination (TPHA), fluorescent treponemal antibody absorption test (FTA-Abs) and the 19-S-(IgM)-FTA-Abs-test. Cerebrospinal fluid (CSF) examination at that time revealed $33 / \mathrm{mm}^{3}$ white blood cells and a total protein of $1.924 \mathrm{~g} / \mathrm{l}$, and there were positive results in TPHA and FTA-A. Furthermore, oligoclonal bands were found in CSF after isoelectric focusing at pH 8.65. Clinical 
neurophysiological examinations revealed no abnormalities in EEG, visual evoked potentials (VEP), median nerve SEP, cranial and thoraco-lumbar computertomographic scans.

On the basis of these findings the diagnosis of an inflammation of the central nervous system (CNS) of syphilitic aetiology was made, ${ }^{910}$ although the history revealed no primary infection. Penicillin treatment with 1 million IE (IM) per day was given. After 30 days of treatment sensory functions and objective signs were improved (CSF cells: $14 / \mathrm{mm}^{3}$; protein $850 \mathrm{mg} / \mathrm{l}$ ). During his stay in the hospital, two neurophysiological recording sessions were carried out within 5 days. After 6 weeks in hospital the patient was discharged.

\section{Neurophysiological examination}

The marked delay in pain perception led to a study of cutaneous pain sensitivity by means of evoked cerebral potentials. For this purpose the hairy skin was stimulated at different dermatomal levels both with electrical stimuli via a stimulus isolation unit, and with radiant heat pulses generated by a high power $\mathrm{CO}_{2}$ laser stimulator. ${ }^{11}$ In healthy subjects the standard laser stimulus ( $20 \mathrm{~ms}, 5 \mathrm{~mm}$ diameter) is painful above $10 \cdot 6 \mathrm{~W} \pm 4 \mathrm{~W}$. With standard electrical pain stimuli ( $200 \mathrm{~Hz}$ rectangular, $20 \mathrm{~ms}$ duration), mean pain threshold in healthy subjects is $5.2 \pm 1.1 \mathrm{~mA}$. $^{12}$

We applied laser stimuli of $14 \mathrm{~W}$ to the dorsum of the patient's left hand (dermatomes $\mathrm{C} 7 / \mathrm{C} 8$ ), the abdominal skin (dermatome L1, left side), below the left knee (dermatome L4) and to the dorsum of the left foot (dermatome L5). In addition, electrical stimuli of $10 \mathrm{~mA}$ were given to the patient's left hand and left foot. In each recording block 40 stimuli of one kind were given with randomised interstimulus intervals between 20 and $40 \mathrm{~s}$.

EEG was recorded from vertex $\left(C_{z}\right.$, according to the International 10-20 system) with reference to linked earlobes (Beckman Accutrace, system bandpass 0.3-30 Hz). For artifact control the vertical component of the electro-oculogram was recorded between upper and lower orbital rims. EEG records contaminated by blinks or gross eye movements were discarded. Both signals were digitised on-line (sampling rate $100 \mathrm{~Hz}$ ). For each recording block a latency corrected average of the non-contaminated EEG segments $(500 \mathrm{~ms}$ before to $2000 \mathrm{~ms}$ after stimulus) was calculated. ${ }^{13}$

\section{Results}

The results of the SEP measurements in the two sessions are summarised in the figure. In the first session, the left hand and the left foot were studied with electrical and with laser pulses. The evoked response after electrical stimulation of the hand (upper left) shows the well known late vertex potential with a negative component at $160 \mathrm{~ms}(\mathrm{~N} 160)$ and a positivity at 280 $\mathrm{ms}$ (P280). Those responses have been observed in many similar experiments with healthy subjects. ${ }^{14}$ Quite similar are the responses to electrical stimulation of the foot (lower left). The vertex negativity appears at $180 \mathrm{~ms}$ followed by the positive component at $290 \mathrm{~ms}$. The slightly longer latencies may be attributed to the different conduction distances. Thus, the electrical stimulation did not reveal any differences in SEP, whether applied to skin areas with normal or with altered pain sensitivity.

In contrast, noxious heat stimuli exhibited distinct differences of SEP latencies between those skin areas with normal and those with delayed pain sensitivity. Stimulation of the hand yielded evoked responses in the normal latency range (N250, P370; upper right). Stimulation of the foot, where pinpricks were only perceived after a delay of approximately one second, led to evoked responses with prolonged latencies in the range of ultralate potentials (N1300, P1420; lower right). In healthy subjects, on the other hand, the latency differences of these ultralate components between upper and lower limb is about $200 \mathrm{~ms}^{13}$

In order to investigate the stability of these ultralate potentials, another session was performed 5 days later, applying only laser stimuli to four different dermatomal levels. Stimulation of the unaffected abdominal skin led to responses in the normal range for late components (N200, P310; second row left). After stimulation at the knee, the vertex negativity appeared at $1190 \mathrm{~ms}$ and the positivity at $1290 \mathrm{~ms}$ (third row left). The response to foot stimulation corresponded to the results of the first session. Thus, in dermatomes below $\mathrm{L} 2$ the sensory dysfunction with delayed pain perception could be documented by a prolonged latency of the SEP components after laser stimulation, whereas conventional electrical stimulation was not able to reflect the delayed pain perception in those dermatomes.

\section{Discussion}

Clinical studies have consistently shown that abnormal somatosensory evoked cerebral potentials are associated with disorders of joint position, touch, vibration and stereognosis. But no SEP abnormalities are seen in neurological disorders affecting only pain or temperature sensations (for example references 15 , 16). These clinical SEP examinations utilise electrical nerve stimulation, and the investigated latency range of cerebral potentials lies between $15 \mathrm{~ms}$ and $100 \mathrm{~ms}$ (for review see reference 5).

In contrast, event related somatosensory cerebral potentials with latencies of more than $100 \mathrm{~ms}$ after somatosensory stimulation have not routinely been used for clinical purposes, although experimental results with healthy subjects describe changes of peakto-peak amplitudes in parallel to the subjective pain estimation after painful electrical skin shocks. ${ }^{17}$ Especially in the evaluation of drug effects these late pain related potentials have been quite useful, ${ }^{1418}$ because they are thought to reflect cortical information processing. However, most of these results were obtained utilising electrical skin shocks. 


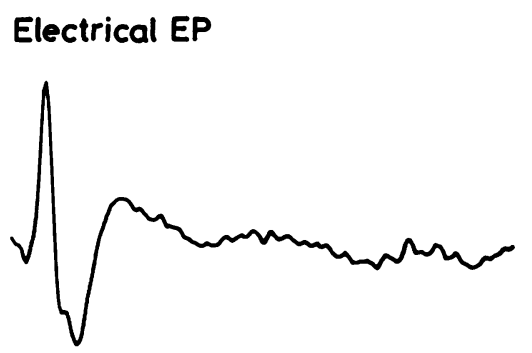

Neurosyphilis

48 year old man Spinal ataxia, numbness of the feet, delayed pain perception below segment $L 2$

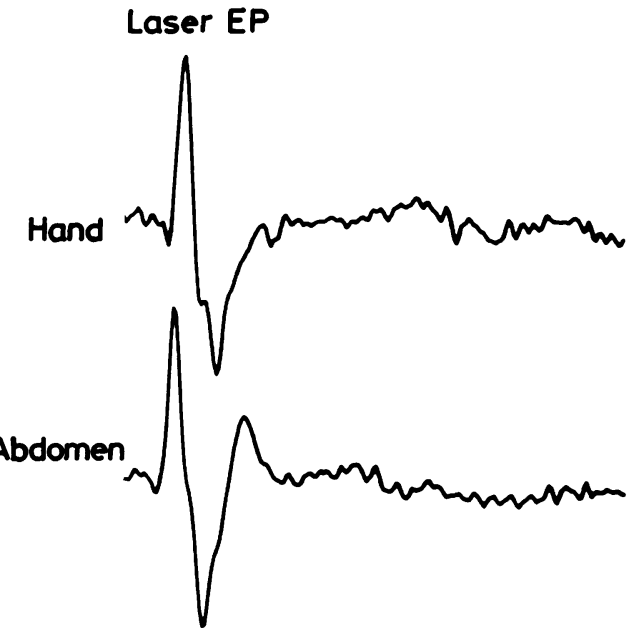

Knee mhannm

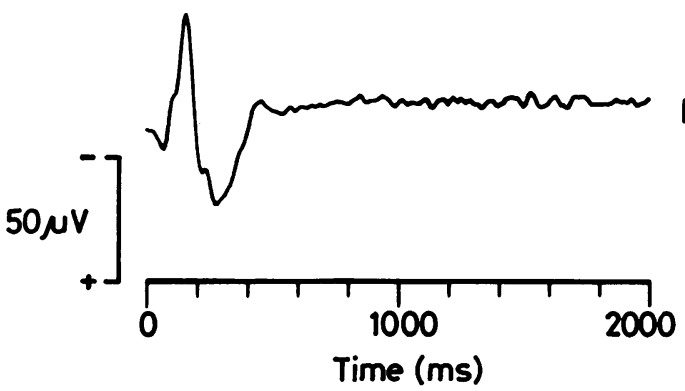

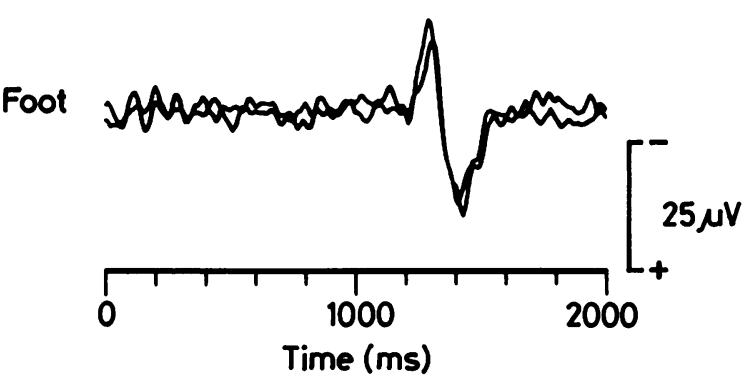

Time (ms)

Fig Evoked cerebral potentials due to electrical ( $10 \mathrm{~mA}$, left) and laser stimuli (14 W, right) in a case of neurosyphilis with delayed pain perception below dermatome L2. The measurements with laser stimulation of the foot were done twice with a five-days interval. Conventional electrical stimuli did not differentiate between affected and unaffected stimulus sites. In contrast, after laser stimulation of unaffected areas (hand and abdomen), evoked responses in the normal latency range appeared, whereas after stimulation of affected dermatomes (knee and foot) the latencies were prolonged by about one second. Latency corrected averages over 40 stimulus repetitions. Vertex versus linked earlobes recording, negativity plotted upward.

Experimental studies with healthy human subjects have repeatedly demonstrated event related potentials in response to noxious radiant heat stimuli produced by $\mathrm{CO}_{2}$-laser. ${ }^{1319}$ After laser stimulation, late SEP components (about $300 \mathrm{~ms}$ latency) correspond to A-delta-fibre mediated pain. ${ }^{20} \mathrm{~A}$ pressure block of the peripheral nerve leads to preferential suppression of A-delta-fibre conduction. In those experiments, ultralate components (about $1300 \mathrm{~ms}$ latency) are found which are correlates of $\mathrm{C}$-fibre mediated pain. ${ }^{13}$ To date, ultralate SEP have been demonstrated only after suppression of A-fibre conduction by preferential nerve block. However, the cerebral potentials seen in our patient with pathological delay of pain perception due to a spinal process are very similar to those found in blocking experiments, although the underlying mechanisms may be of quite different nature.

The reason for delayed pain perception in neurosyphilis is still unknown. A deficiency in synaptic transmission within the dorsal horn, as implied in both painful sensory disturbances and pain alleviation, ${ }^{21-23}$ may cause delayed temporal summation and thus an increase in latency of central projection. Another possible mechanism of the pathological delay 
may be an impairment of A-delta-fibres in the dorsal root entry zone, where A- and C- fibres lie separately, ${ }^{24}$ or of the corresponding neurons in the dorsal horn, as already discussed by Lewis and Pochin. ${ }^{1}$ In this case, only the slower conducting fibres would project neural information to the brain.

The present findings cannot reveal the cause of the pathological pain perception in neurosyphilis. But they demonstrate that by means of modality specific stimulation it is possible to assess disorders of pain perception by means of somatosensory evoked potentials. Up to now, these methods have hardly been introduced into clinical routine diagnostics. Lower costs of laser stimulation devices and less difficult handling may lead to a broader application, for example in syringomyelia or the syndrome of the anterior spinal artery as well as in other diseases accompanied by dissociated sensory loss.

This study was supported by the Deutsche Forschungsgemeinschaft (Sonderforschungsbereich 115; Schwerpunktprogramm Nociception und Schmerz).

\section{References}

1 Lewis T, Pochin EE. The double pain response of the human skin to a single stimulus. Clin Sci 1937;3:67-76.

2 Melzack R. The Puzzle of Pain. New York: Basic Books, 1973:139-40.

3 Burke JM, Schaberg DR. Neurosyphilis in the antibiotic era. Neurology 1985;35:1368-71.

4 Jones SJ. Somatosensory evoked potentials: the abnormal waveform. In: Halliday AM, ed. Evoked Potentials in Clinical Testing. Edinburgh: Churchill Livingstone, 1982:429-70.

5 Chiappa KH. Interpretation of abnormal short-latency somatosensory evoked potentials. In: Halliday AM, Butler SR, Paul R, eds. A Textbook of Clinical Neurophysiology, New York: Wiley \& Sons, 1987:343-81.

6 Mor J, Carmon A. Laser emitted radiant heat for pain research. Pain 1975;1:233-7.

7 Treede RD, Bromm B. Reliability and validity of ultra late cerebral potentials in response to C-fibre activation in man. In:
Dubner R, Gebhart GF, Bond MR, eds. Proceedings of the Vth World Congress on Pain. Amsterdam: Elzevier 1988: 567-73.

8 Arendt-Nielsen L, Bjerring P. Sensory and pain threshold characteristics to laser stimuli. J Neurol Neurosurg Psychiatry 1988;51:35-42.

9 Storm-Mathisen A. Syphilis. In: Vinken PJ, Bruyn GW, eds. Handbook of Clin Neurol Vol 33, Amsterdam: North-Holland, 1978:337-94.

10 Simon RP. Neurosyphilis. Arch Neurol 1985;42:606-13.

11 Biehl R, Treede RD, Bromm B. Pain ratings of short radiant heat pulses. In: Bromm B, ed. Pain Measurement in Man, Amsterdam: Elsevier, 1984:397-408.

12 Bromm B, Meier W, Scharein E. Antagonism between tilidine and naloxone on cerebral potentials and subjective pain ratings in man. Eur J Pharmacol 1983;87:431-9.

13 Bromm B, Treede RD. Human cerebral potentials evoked by $\mathrm{CO}_{2}$ laser stimuli causing pain. Exp Brain Res 1987;67:153-62.

14 Bromm B, Meier W, Scharein E. Imipramine reduces experimental pain. Pain 1986;25:245-57.

15 Halliday AM, Wakefield GS. Cerebral evoked potentials in patients with dissociated sensory loss. J Neurol Neurosurg Psychiatry 1963;26:211-9.

16 Giblin DR. Somatosensory evoked potentials in healthy subjects and in patients with lesions of the nervous system. Ann NY Acad Sci 1964;112:93-142.

17 Chudler EH, Dong WK. The assessment of pain by cerebral evoked potentials. Pain 1983;16:221-44.

18 Chapman CR, Colpitts YM, Benedetti C, Butler S. Event-related potential correlates of analgesia; comparison of fentanyl, acupuncture and nitrous oxide. Pain 1982;14:327-37.

19 Bjerring P, Arendt-Nielsen L. Argon laser induced single cortical responses: a new method to quantify pre-pain and pain perceptions. J Neurol Neurosurg Psychiatry 1988;51:43-9.

20 Kenton B, Coger R, Crue B, Pinsky J, Friedman Y, Carmon A. Peripheral fibre correlates to noxious thermal stimulation in humans. Neurosci Lett 1980;17:301-6.

21 Tasker RR, Tsuda T, Hawrylyshyn P. Clinical neurophysiological investigation of deafferentiation pain. In: Bonica JJ, ed. Adv Pain Res Ther, Vol. 5, New York: Raven Press, 1978:713-38.

22 Rémillard GM, Robitaille Y, Bertrand G. Douleur chronique et fente syringomyélique des cornes postérieures de la moelle épinière. Rev Neurol (Paris) 1985;141:386-90.

23 Saris SC, Iacono RP, Nashold BS. Dorsal root entry zone lesions for post-amputation pain. $J$ Neurosurg 1985;62:72-6.

24 Sindou M, Quoex C, Baleydier C. Fiber organization at the posterior spinal cord-rootlet junction in man. J Comp Neurol 1974;153:15-26. 